\title{
Erratum: The large-scale nebular pattern of a superwind binary in an eccentric orbit
}

Hyosun Kim, Alfonso Trejo, Sheng-Yuan Liu, Raghvendra Sahai, Ronald E. Taam, Mark R. Morris, Naomi Hirano \& I-Ta Hsieh

Nature Astronomy 1, 0060 (2017); published 1 March 2017; corrected 6 March 2017.

The Supplementary Information originally published did not include Supplementary Video 1 and its associated caption. These are now included in the updated Supplementary Information. 